\title{
Engineered protein-protein interaction regulators for therapeutic applications
}

Bar-Ilan University, Israel

https://doi.org/10.17952/35EPS.2018.047

Protein-protein interactions (PPIs) are intimately involved in almost all biological processes, including interand intracellular signal transduction,gene expression, cell proliferation and apoptosis. Therefore, they are important phenomena in basic research and promising targets for treating human disease. Nevertheless, targeting PPIs is challenging, as PPI interfaces are large, flat, and are usually endowed with a significant degree of conformational flexibility. Peptides are ideal candidates for targeting PPIs as they have demonstrated high conformational flexibility, increased selectivity, and are generally inexpensive. Moreover, peptide limitations, such as poor stability and inefficient crossing of cell membranes, can be overcome using peptidomimetics (modified peptides) [1-4].

Here we combine two techniques for the development of peptidomimetics. The first is an approach for the identification of the two critical PPI interfaces. We developed novel algorithms to detect specific PPI interfaces between a vital protein and only one of its many partners, using rational design, based on unique interaction sites (Fig. 1) [5-10]. A second technique, backbone cyclization, is an already established approach used for the development of peptidomimetics that retain the functional groups of the substrate side chain residues essential for their bioactivity. We thus convert active sequences into peptidomimetics while retaining their conformational flexibility (Fig. 2) [11, 12]. Together, these techniques allow the development of selective inhibitors of specific binding sites that are metabolically stable and highly active.

One example of inhibition of specific PPIs is the selective inhibition of the phosphorylation of pyruvate dehydrogenase kinase $(\mathrm{PDK})$ by delta protein kinase $\mathrm{C}(\delta \mathrm{PKC})$, which demonstrated its correlation with cellular processes following cardiac ischemia. Since $\delta$ PKC phosphorylates many substrates, the extent to which this specific phosphorylation is critical for cardiac damage could not be previously explored. We developed an inhibitor of the $\delta$ PKC/PDK PPI, $\psi \mathrm{PDK}$, which selectively inhibits PDK phosphorylation. In animal models of heart attack, $\psi$ PDK reduced infarct size by $55 \%$ and tripled ATP levels. $\psi$ PDK was found to be highly active (EC50 $5 \mathrm{nM}$ ) and specific, and did not affect the phosphorylation of other $\delta$ PKC substrates even at $1 \mu \mathrm{M}[13$, $14]$.

A second example of specific PPI inhibition involves proteins that regulate mitochondrial fission, dynamin-related protein 1 (Drp1) and mitochondrial outer membrane protein, Fis1. Excessi ve mitochondrial fission results in oxidative stress and contributes to the pathology of neurodegenerative diseases. We developed a selective inhibitor of the Drp1/Fis1 PPI, p110. The selective inhibition reduced ROS production,improved mitochondrial membrane potential and reduced apoptosis. Our overall findings demonstrated that P110 prevents mitochondrial fragmentation and cell death under pathological conditions [15].

Inhibition of $\delta$ PKC/PDK and Drp1/Fis1 PPIs are both examples of specific and selective inhibition by rationally designed peptidomimetics. These novel, highly selective PPI inhibitors may be used as lead compounds for therapeutic applications. Furthermore, similar approaches can be applied for the development of various other specific PPI regulators. 


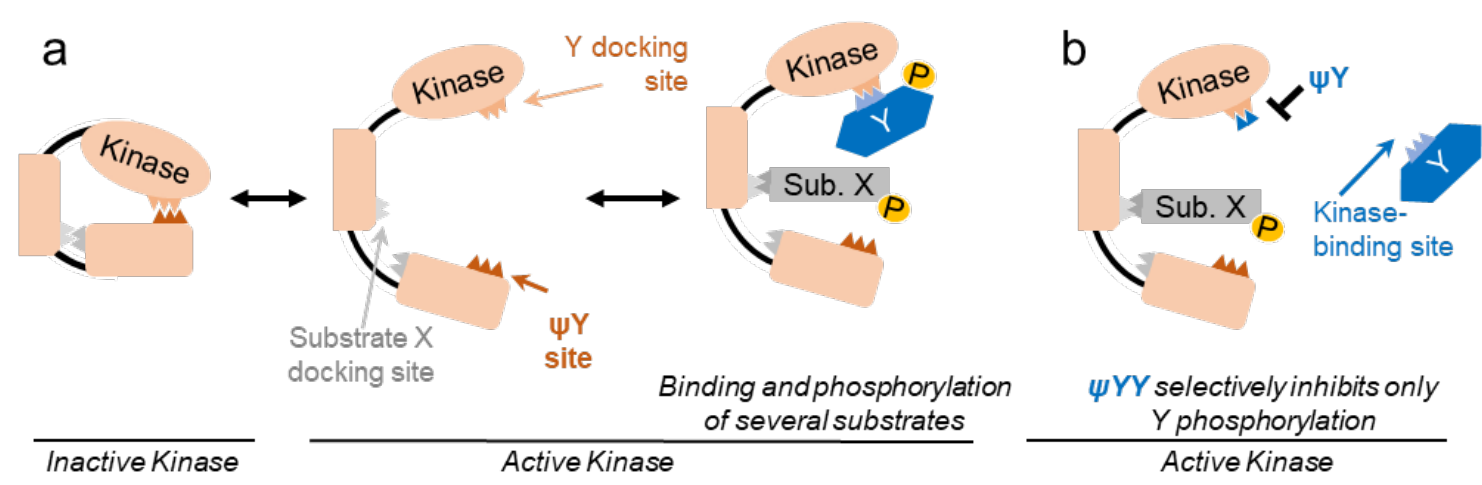

Figure 1: A scheme representing the design of a PPI modulator that is selective for the interaction between one substrate and a multi-substrate kinase. (a) In its inactive state (aka 'closed', or auto-inhibited conformation) (left), the substrate-docking site on the kinase forms an intramolecular interaction with a sequence similar to the kinase-binding site on the substrate, termed the pseudo-specific substrate site ( $\psi Y$, brown). Upon activation, the kinase undergoes a conformational change, disrupting the intramolecular interaction and exposing its active site. As a result, the substrate-specific docking sites are available for binding (shown are docking sites for $Y$ and substrate X (Sub. X) on the kinase, two of several protein substrates of this kinase). Specific PPIs between the kinase and its substrates results in substrate phosphorylation (P). (b) A peptide corresponding to the kinase-like sequence on $Y, \psi Y$ (blue), is a competitive inhibitor for docking to and phosphorylation of $Y$ by the kinase without inhibiting docking and phosphorylation of other kinase substrates (e.g. Sub. X).

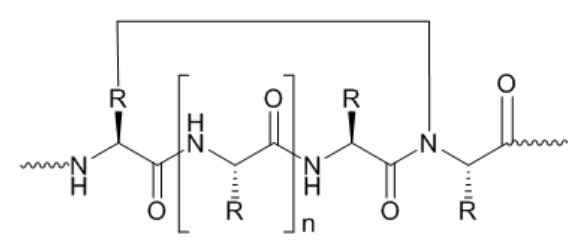

a. N-backbone to side chain

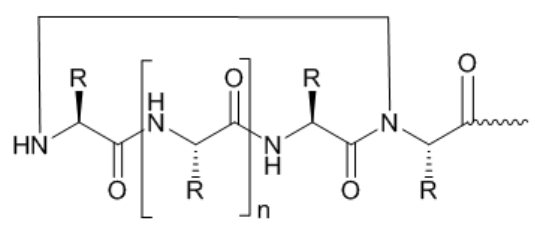

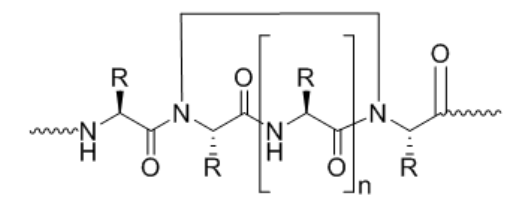

b. $\mathrm{N}$ to $\mathrm{N}$-backbone

c. N-backbone to head/tail

Figure 2: Backbone cyclization approach for alternative peptide cyclization strategies. Backbone cyclization is a technique that enables development of cyclic peptides without utilizing the residues that are part of the natural linear peptide, which may be essential for the biological activity of the peptide. In backbone cyclization, a building unit composed of a functional atom with an orthogonal protecting group, covalently bonded via spacer to the backbone, is used to form a cyclic peptide from a linear parent molecule. The main advantage of this method is that the cyclization linkage is formed between backbone atoms and not the atoms of the side chain functional groups, which are typically critical for binding and biological function. There are eight modes of cyclization amenable to backbone cyclization technique, including four natural modes: head-to-tail (C-terminus to $N$-terminus), side chain-to-tail, side chain-to-side chain, and side chain-to-head; and four modes of backbone cyclization: backbone-to-side chain (a), backbone-to-backbone (b), and backbone-to-N or -to-C terminus (c).

\section{References}

[1] Rubin, S.; Qvit, N., Cyclic peptides for protein-proteininteraction targets: Applications to human disease. Critical Reviews in Eukaryotic Gene Expression, 2016, 26, (3), 199-221.

[2] Cunningham,A.D.; Qvit, N., Specific kinase inhibition using peptides to target kinase-substrate docking. Chimica Oggi/Chemistry Today, 2016, 34, (5), 22-25. 
[3] Qvit, N.; Rubin, S.J.S.; Urban, T.J.; Mochly-Rosen, D.; Gross, E.R., Peptidomimetic therapeutics: Scientific approaches and opportunities. Drug Discovery Today, 2017, 22, (2), 454-462.

[4] Cunningham, A.D.; Qvit, N.; Mochly-Rosen, D., Peptides and peptidomimetics as regulators of proteinprotein interactions. Current Opinion in Structural Biology, 2017, 44, 59-66.

[5] Qvit, N.; Crapster, J.A., Peptides that target protein-proteininteractions as an anti-parasite strategy. chimica Oggi / CHEMISTRY today, 2014, 32, (6), 62-66.

[6] Qvit, N.; Joshi, A.U.; Cunningham, A.D.; Ferreira, J.C.; Mochly-Rosen, D., GAPDH (glyceraldehyde3-phosphate dehydrogenase) protein-protein interaction inhibitor reveals a non-catalytic role for GAPDH oligomerization in cell death. Journal of Biological Chemistry, 2016, 291, (26), 13608-13621.

[7] Qvit, N.; Kornfeld, O.S.; Mochly-Rosen, D., Engineered substrate-specific delta PKC antagonists to enhance cardiac therapeutics. Angewandte Chemie, International Edition in English, 2016, 55, (50), 15672-15679.

[8] Qvit, N.; Schechtman, D.; Pena, D.A.; Berti, D.A.; Soares, C.O.; Miao, Q.; Liang, L.; Baron, L.A.; Teh-Poot, C.; Martínez-Vega, P.; Ramirez-Sierra, M.J.; Churchill, E.; Cunningham, A.D.; Malkovskiy, A.V.; Federspiel, N.A.; Gozzo, F.C.; Torrecilhas, A.C.; Manso Alves, M.J.; Jardim, A.; Momar, N.; Dumonteil, E.; MochlyRosen, D., Scaffold proteins LACK and TRACK as potential drug targets in kinetoplastid parasites: Development of inhibitors. InternationalJournal for Parasitology: Drugs and Drug Resistance, 2016, 6, 74-84.

[9] Qvit, N.; Kornfeld, O.S., Development of a backbone cyclic peptide library as potential antiparasitic therapeutics using microwave irradiation. Journal of Visualized Experiments, 2016, (107), e53589.

[10] Franco, A.; Kitsis, R.N.; Fleischer, J.A.; Gavathiotis, E.; Kornfeld, O.S.; Gong, G.; Biris, N.; Benz, A.; Qvit, N.; Donnelly, S.K.; Chen, Y.; Mennerick, S.; Hodgson, L.; Mochly-Rosen, D.; Dorn, G.W., Correcting mitochondrial fusion by manipulating mitofusin conformations. Nature, 2016, 540, (7631), 74-79.

[11] Rubin, S.J.; Tal-Gan, Y.; Gilon, C.; Qvit, N., Conversion of Protein Active Regions into Peptidomimetic Therapeutic Leads Using Backbone Cyclization and Cycloscan-How to Do it Yourself! Current Topics in Medicinal Chemistry, 2018, 18, (7), 556-565.

[12] Rubin, S.J.S.; Qvit, N., Backbone-Cyclized Peptides: A Critical Review. Current Topics in Medicinal Chemistry, 2018, 18, (7), 526-555.

[13] Qvit, N.; Mochly-Rosen, D., Highly specific modulators of protein kinase C localization: Applications to heart failure. Drug Discovery Today: Disease Mechanisms, 2010, 7, (2), e87-e93.

[14] Qvit, N.; Disatnik, M.H.; Sho, J.; Mochly-Rosen, D., Selective phosphorylation inhibitor of delta protein kinase C-pyruvate dehydrogenase kinase protein-proteininteractions: Application for myocardial injury in vivo . Journal of the American Chemical Society, 2016, 138, (24), 7626-7635.

[15] Qi, X.; Qvit, N.; Su, Y.C.; Mochly-Rosen, D., A novel Drp1 inhibitor diminishes aberrant mitochondrial fission and neurotoxicity. Journal of Cell Science, 2013, 126, (Pt 3), 789-802. 